\title{
Ueber die quantitative Bestimmung des Harnindicans nach Wang-Obermayer.
}

\author{
Von \\ Jac. Bouma.
}

(Aus dem physiologischen Laboratorium der Universität in Utrecht.)

(Der Redaction zugegangen am 8. Mai 1899.)

Nachdem im August 1898 die Arbeit von Wang ${ }^{1}$ ) über die quantitative Bestimmung des Harnindicans erschienen war, habe ich sofort nach diesem Verfahren viele Bestimmungen gemacht und bei verschiedenen Proben desselben Harnes gut. übereinstimmende Resultate erhalten. Bei der Titrirung befolgte ich die Angabe Wang's, dass man als Endreaction nicht eine rothe, sondern eine schwach gelbliche Farbe der Titrirlösung zu betrachten habe. Die Indicanmengen, berechnet als Indigotin, betrugen bei normalem menschlichen Harn ca. 5 mgr. pro Tag und stiegen in pathologischen Fällen iffters bis zu 18 mgr. Die Bestimmungen der gepaarten Schwefelsäure standen hiermit im Einklang, insofern als das Verhältniss zwischen Indoxylschwefelsäure und der totalen gepaarten Schwefelsäure, sowohl bei indicanreichem als auch bei indicanarmem Harn, ziemlich constant war. Bisweilen trat beim Eingiessen des mit Schwefelsäure behandelten Indigos in Wasser eine missfarbene Titrirlösung auf, doch in weitaus den meisten Fällen eignete sich die erhaltene Lösung ganz gut zur Titration.

Als nun Obermayer ${ }^{2}$ ) mittheilte, man sollte die röthlich-braunen Farbstoffe, die sich nach dem Verdunsten des Chloroforms als Belag auf das Indigoblau absetzen, mit 45\% igem Alkohol ausziehen und dann das reine Indigoblau als Disulfo-

1) Hoppe-Seyler's Zeitschr. f. physiol. Chemie, Bd. XXV, S. 406.

2) Hoppe-Seyler's Zeitschr. f. physiol. Chemie, Bd. XXVI, S. 427 und Wiener klin. Rundschau 1898, Nr. 34, S. 1. 
säure zur Titration bringen, habe ich vergleichende Versuche nach beiden Methoden gemacht und bei Benutzung desselben Harns ganz verschiedene Resultate erhalten. Das eine Mal waren die Quantitäten Indigotin nach beiden Methoden annähernd gleich, ein anderes Mal war ein beträchtlicher Unterschied vorhanden. Durchschnittlich fand ich nach Obermayer $20 \%$ Indigotin weniger als nach Wang. Dieser Unterschied kam natürlich daher, dass ich nach Wang auch die röthliche Farbe mit Kaliumpermanganat zum Schwund brachte.

Neuerdings erschien eine andere Mittheilung von Wang, ${ }^{1}$ ) worin er zur Auswaschung der röthlichen Farbstoffe ein besseres Mittel empfahl, eine Mischung von Aetheralkohol und Wasser zu gleichen Theilen.

Nach dieser Behandlung bringt man nun wirklich eine prachtvolle, rein blaue Indigotindisulfosäurelösung zur Titration, jedoch wird die Menge des Indigotins, wie auch Wang selbst mittheilt, hierdurch weiter um ca. 10\% herabgesetzt, so dass mit den ersten 20\% (Waschung nach Obermayer) ein Gesammtverlust von etwa $30 \%$ entsteht. Wang's Waschungsmethode ergibt also titrimetrisch durchschnittlich $30 \%$ weniger Indigotin als sein ursprüngliches Verfahren.

Bei diesen sogenannten Reinigungen hat man nun aber die gelösten Farbstoffe (von $\mathrm{W}$ ang kurzweg Verunreinigungen genannt) einfach entfernt, ohne sich zu fragen, was sie eigentlich sind oder was sie mit der Indicanbestimmung $\mathrm{zu}$ thun haben können. Anfangs habe ich mir die Sache so vorgestellt, es werde sich bei der Behandlung des Chloroformrückstandes mit Schwefelsäure ein Theil des Indigotins in die Monosulfosäure (die purpurrothe Phoenicinschwefelsäure) umbilden. Da jedoch in den meisten Fällen nach dem Verdunsten des Chloroforms die rothen Farbstoffe sich deutlich auf die blauen absetzten, bevor die Behandlung mit Schwefelsäure geschah, musste ich hiervon absehen. Wir werden es hier vielmehr mit einer theilweisen Umbildung des Indoxyls in Indigoroth und Indigobraun $\mathrm{zu}$ thun haben. Bekanntlich wird auch bei der Bereitung von

1) Hoppe-Seyler's Zeitschr. f. physiol. Chemie, Bd. XXVII, S. 135. 
Indigotin aus Pflanzenindican neben dem Indigoblau immer eine gewisse Quantität Indigoroth und Indigobraun gebildet, bei der Ammoniakmethode sogar bis zu 14\%. Da nun der Mutterstoff des Indigo im Pflanzenindican Indoxyl in der Form eines. Glucosids, im Harnindican Indoxyl in der Form eines Schwefelsäureesters ist, da ferner bei beiden zuerst das Molekül einer hydrolytischen Spaltung unterworfen wird, worauf bei beiden die Oxydation des Indoxyls zu Indigo erfolgt, so wird es nichts Aussergewöhnliches sein, dass bei beiden Processen ähnliche Körper gebildet werden. Ich glaube dies um so eher, als. ich in demselben Harn bei gleicher Behandlung bald mehr, bald weniger Rothbildung fand. So erklären sich auch die Differenzen, die ich beim Vergleich von Wang's erster Methode und der von Obermayer (Waschung mit 45\% igem Alkohol) erhielt. Es handelt sich hier also nicht um Skatol- oder andere Farbstoffe, sondern um Modificationen des Indigos. Da. diese nun in fortwährend wechselnden Quantitäten, bis zu $30 \%$, gebildet werden, so muss man sie bei der Indicanbestimmung ganz genau mitberechnen. Zunächst muss aber bewiesen werden, dass der rothbraune Belag des Chloroformrückstandes wirklich Indigofarbstoffe sind, da das bisher Erwähnte nur Hypothese ist.

Bevor es mir gelang, die völlige Trennung der verschiedenen Indigofarbstoffe zu bewerkstelligen, habe ich nur auf Roth und Blau reagirt. Mir bekannte Reactionen auf Mischungen von Indigoroth und blau waren folgende:

Indigoroth ist löslich in Aether, Alkohol und Chloroform, während Indigoblau nur in Chloroform löslich ist.

Löst man eine Mischung von Indigoroth und Indigoblau auf dem Wasserbade in Eisessig und lässt danach ruhig abkühlen und spontan verdunsten, dann setzt sich das Blau auf dem Boden der Schale ab, während das Roth in Lösung bleibt.

Titrirt man eine Mischung von Indigoroth und -blau als Disulfosäuren mit Kaliumpermanganat, dann wird das Blau sofort enitfärbt, während das Roth nur sehr langsam oxydirt wird. Nach dem Verschwinden der blauen Farbe der Titrirlösung tritt also die rothe auf, die langsam schwindet. 
Diese drei Reactionen stimmen vollkommen überein mit dem Verhalten des aus Harn erhaltenen Chloroformrückstandes bei der Untersuchung nach dem ursprünglichen Verfahren von Wang.

Bis jetzt hatte ich Roth und Blau nur mittelst kalten Alkohols von einander getrennt und daher vom Indigobraun keine Spur entdeckt. Nur wies die rothbraune Farbe des Alkoholextractes und des Belags des Chloroformrückstandes auf die Anwesenheit des Braun neben dem Roth hin, und alle Autoren sprechen daher imme von rothbraunen Farbstoffen. Als ich nun den Rückstand vorsichtig mit Aether übergoss, löste sich darin das Indigoroth mit der ihm eigenen schönen purpurrothen Farbe, während auf dem Indigoblau ein brauner Belag blieb. Dieser Belag löste sich in kaltem Alkohol mit schöner kaffeebrauner Farbe, worauf das reine Blau auf dem Boden der Schale zurückblieb, das sich nun mit rein blauer Farbe in Chloroform löste. Diese Trennung der aus Harn erhaltenen Farbstoffe in Roth, Braun und Blau ist sehr scharf, und man erhält schöne, reinfarbige, klare Lösungen, die zur spectroskopischen Untersuchung geeignet sind.

Hierbei fand ich, dass die Spectra der von mir aus Harn erhaltenen Indigofarbstoffe ganz übereinstimmen mit denen von reinem Pflanzenindigoroth, -braun und -blau.

Die pflanzlichen Indigofarbstoffe habe ich aus käuflichem Indigo nach folgendem Verfahren erhalten: Indigo wird mit verdünnter Schwefelsäure öfters längere Zeit gekocht, bis das Filtrat nahezu farblos ist, und der Rückstand mit Wasser ausgewaschen, bis das Filtrat keinen Niederschlag mit Baryumchlorid mehr gibt. Der Indigo ist dann von den humusartigen Bestandtheilen und vom Indigoleim befreit, während er von verdünnter Säure nicht angegriffen wird. Der Rückstand wird jetzt mit Chloroform in einem Kolben mit Rückflusskühler gekocht und die erhaltene Lösung in einer Schale verdunstet, wobei sich nun nacheinander Blau, Braun und Roth absetzen, wie bei den aus Harn erhaltenen Lösungen. Die verschiedenen Farben lassen sich dann auf bekannte Weise mit Aether, Alkohol und Chloroform trennen. Für spectroskopische 
Zwecke ist dieses Verfahren um so mehr zu empfehlen, da man reines Indigoroth erhält, denn das Braun bildet eine schützende Decke über dem Blau, welch letzteres spurweise in Aether löslich ist, während das Indigobraun darin absolut unlöslich ist. Nach anderen Methoden löst man im Aether immer eine Spur Blau auf, wodurch die purpurrothe Farbe einen Stich ins Violette bekommt; solche Lösungen zeigen spectroskopisch immer eine Andeutung von Indigoblaustreifen links von D. Auf die genannte Weise bekommt man aber die reinen Lösungen der drei pflanzlichen Indigofarbstoffe zur spectroskopischen Untersuchung.

Was nun die Spectra dieser Lösungen betrifft, so habe ich schon erwähnt, dass die der Pflanzen- und der Harnindigofarbstoffe vollkommen identisch sind. Beide Indigoblaulösungen in Chloroform zeigen einen breiten Absorptionsstreifen zwischen d und D; der Streifen setzt mit 630 ziemlich scharf ein, läuft über $\mathrm{D}$ weg, nimmt von 590 bis 570 langsam ab und besitzt seine grösste Intensität bei 605 . Beide Indigobraunlösungen in Alkohol-zeigen gar keine Absorptionsstreifen; sie verdunkeln nur das ganze Spectrum. Beide Indigorothlösungen in Aether zeigen in verdünntem Zustande einen Absorptionsstreifen in gelbgrün, anfangend zwischen $D$ und $\mathrm{E}$ bei 560 , der über $\mathrm{E}$. abklingend wegzieht. Bei etwas stärkeren Lösungen fängt die Absorption kurz hinter D an und das ganze Grün wird bis hinter F absorbirt; ausserdem ist das ganze übrige violette Ende des Spectrums etwas verdunkelt. Bei ziemlich concentrirten Lösungen fängt die Absorption bei $\mathrm{D}$ scharf an und läuft bis kurz an G (bis ungefähr 450), während jetzt auch das ganze violette Ende des Spectrums sehr verdunkelt ist.

Ausser diesen genannten Uebereinstimmungen zwischen Pflanzen- und Harnindigofarbstoffen muss noch erwähnt werden, dass beide Indigobraunarten löslich sind in verdünnter Kaliund Natronlauge, worin Roth und Blau unlöslich sind.

Die Variabilität der auftretenden Mengen der Indigofarbstoffe aus Harn muss vielleicht auf Rechnung von Polymerie gesetzt werden. Die procentische Zusammensetzung der Indigomodificationen ist dieselbe, dagegen zeigen Löslichkeit und 
Sublimationstemperatur ein ganz eigenthümliches Verhalten. Mit dem Abnehmen der Löslichkeit steigt die Sublimationstemperatur; erstere nimmt von Roth nach Blau ab, während letztere von Roth nach Blau steigt. Dieses Verhalten bei gleicher elementarer Zusammensetzung macht es nicht unwahrscheinlich, dass wir es hier mit Polymerie zu thun haben, wobei Roth das kleinste und Blau das grösste Indigomolekül haben soll. Es lässt sich auf diesem Wege das Auftreten der wechselnden Quantitäten der Indigomodificationen erklären, indem bei der Oxydation der befreiten Indoxylmoleküle zu. Indigo die gebildeten Moleküle des letzteren auf verschiedenen Polymerisationsstufen stehen bleiben. Für Polymerie spricht auch die theilweise Umbildung von reinem Indigoblau in -roth bei Sublimation und bei Kochen der Chloroformlösung desselben. Auch die schnellere Oxydation der Indigoblaudisulfosäure gegenüber der Sulfosäure des Indigoroths mittelst Kaliumpermanganats steht hiermit im Einklang, indem beim Auseinanderfallen des grossen Indigoblaumoleküls wahrscheinlich viel Energie frei wird, die den Oxydationsprocess beschleunigen kann. Umgekehrt muss bei der Bildung des Indigos aus Indoxyl Energie in Form von Wärme zugeführt werden, wenn man ausschliesslich die höchste Polymerisationsstufe erlangen will. Die bei der Oxydation gebildete Wärme scheint dazu nicht zu genügen, und deshalb bleibt ein Theil auf niedriger Stufe stehen. Entzieht man also Energie durch Abkühlung, so hat man vorzugsweise Indigorothbildung $\mathrm{zu}$ erwarten.

Die Experimente stimmen hiermit vollkommen überein. Eine gewisse Quantität Harn wird mit Bleizuckerlösung gefällt und das klare Filtrat in drei gleiche Portionen getheilt.

Portion I wird in Eis auf $0^{\circ}$ abgekühlt und danach ein gleich grosses Quantum Obermayer's Reagens tropfenweise zugegossen, sodass die Temperatur nicht über $3^{0}$ steigt. Man lässt nun längere Zeit in Eis stehen, da die Oxydation bei $0^{0}$ nur langsam von Statten geht. Portion II wird auf die gewohnte Weise bei Zimmertemperatur behandelt. Portion III wird 10-15 Minuten mit Obermayer's Reagens in einem Wasserbade von ungefähr $45^{\circ}$ erwärmt; die Tempertur darf 
$50^{\circ}$ nicht überschreiten, denn schon beim Siedepunkt von Chloroform scheint sich in Lösung befindliches Indigoblau wieder theilweise $\mathrm{zu}$ depolymerisiren $\mathrm{zu}$ Indigoroth. Erwärmt man kurze Zeit bei Siedehitze, dann wird hauptsächlich Roth gebildet; bei fortgesetztem Kochen wird alles zu höheren Stufen oxydirt und man bekommt keine Spur von Indigo beim Ausschütteln.

Die drei Portionen werden jetzt mit Chloroform ausgeschüttelt, wobei man von Portion I eine rothviolette, von Portion II eine blauviolette und von Portion III eine rein blaue Chloroformlösung bekommt. Da ich öfters bemerkt hatte, dass während des Abdestillirens des Chloroforms bei Siedehitze desselben die blaue Farbe plötzlich einen Stich ins Violette zeigte, ja sogar ins Blauviolette umschlug, kam ich zu der schon erwähnten Hypothese der Depolymerisation und ich habe das Chloroform im Vacuum verdunstet. Spontane Verdunstung im Dunkeln ist auch zweckmässig, doch dauert sie recht lange.

Der Rückstand von Portion I zeigt sich nun auf der weissen Porzellanschale rothviolett, von Portion II blau mit rothbraunem Belag, von Portion III fast rein blau mit sehr geringer Rothfärbung. Durch Behandeln der Rückstände zuerst mit Aether und dann mit kaltem Alkohol werden nun successive Indigoroth und -braun gelöst. Wiewohl man hierbei bemerkt, dass bei Portion III die Bildung dieser Modificationen auf ein Minimum beschränkt ist, so muss ich doch gestehen, dass es mir niemals gelungen ist, allein reines Indigoblau darzustellen.

Die in den Schalen zurückbleibenden Mengen reinen Indigoblaus wưrden nun als Disulfosäuren mit Kaliumpermanganat titrirt, wobei sich ergab, dass für Portion I 3,15, für Portion II 6,55 und für Portion III 7,60 ccm. zur Titration nöthig waren. Die Mengen des Indigoblaus sind natürlich diesen Zahlen proportional.

Diesen Versuch habe ich oft wiederholt, sowohl mit indicanreichem als mit indicanarmem Harn, und immer einen ähnlichen Befund, wenn auch mit etwas anderen Zahlen, constatiren können.

Nach allen diesen Erörterungen glaube ich, dass wir es 
hier unzweifelhaft mit den Modificationen des Indigos zu thun haben, die sich nebeneinander bei der Oxydation des befreiten Indoxyls bilden. Es scheint mir also nicht richtig, bei der Bestimmung des Harnindicans nur auf das Indigoblau Acht zu geben, wie es Obermayer gethan, dem sich Wang in seiner letzten Mittheilung angeschlossen hat. Die rothen und braunen Farbstoffe, welche von diesen Autoren möglichst vollständig entfernt werden, sind nicht etwa vom Skatoxyl herstammende Verunreinigungen, sondern Oxydationsprodukte des Indoxyls. Es ist bis jetzt aber kein Mittel gefunden worden, das gestattet, die Oxydation des Indoxyls so verlaufen zu lassen, dass Blau, Braun und Roth in bestimmtem Verhältniss zu einander gebildet werden. Es ist deshalb nicht richtig, den mittelst Chloroform erhaltenen Indigo mit Alkohol oder Alkoholäther auszuwaschen. Beim Titriren der in Schwefelsäure gelösten Farbstoffe soll solange Kaliumpermanganat zugesetzt werden, als die Flüssigkeit noch eine Spur von einer rothen Farbe zeigt; Erwärmen beschleunigt die Titration des Roths. Solange die rothe Farbe bleibt, ist noch Indigorothdisulfosäure in Lösung; diese rothe Farbe kann nicht vom Kaliumpermanganat herstammen, da diese, auch wenn aller Indigo zu Isatin oxydirt ist, entfärbt wird, indem das Isatin weiter oxydirt wird zu farbloser Anthranilcarbonsäure.

Die Waschungsmethoden würden nur dann brauchbare Resultate liefern, wenn das Mengenverhältniss der auftretenden Modificationen immer constant wäre. Um mich von der Richtigkeit meiner Auffassung zu überzeugen, habe ich 6 Tage lang täglich $1 \frac{1}{2}$ Liter normalen Menschenharn verarbeitet, wobei ich Wang's ursprüngliches Verfahren befolgte, um einen grösseren Mengenunterschied zwischen Totalindigo und reinem Indigoblau zu erzeugen. Ich erhielt daraus im Ganzen $30 \mathrm{mgr}$. Indigo, woraus nach Auswaschen mit Aetheralkohol $20 \mathrm{mgr}$. reines Blau resultirten. Die tägliche Titrirung desselben Harns ohne Waschung ergab ein mit $30 \mathrm{mgr}$. übereinstimmendes Quantum.

Utrecht, Mai 1899. 